\title{
Macro e micro fotogrammetria per la virtualizzazione della laminetta orfica (V-IV a.C.) del Museo Nazionale di Vibo Valentia
}

\author{
Sara Antinozzi \\ Diego Ronchi \\ Salvatore Barba
}

\begin{abstract}
Affrontare la discussione di applicazioni consolidate di fotogrammetria non costituisce in sé un'attività necessariamente innovativa. Meritano, tuttavia, un costante approccio critico le originali implementazioni offerte non solamente dall'evoluzione tecnologica degli strumenti di acquisizione, ma anche dalle sfide poste a noi rilevatori e più in generale alla teoria delle misure. Le nuove opportunità di descrivere e rappresentare la realtà sono prevalentemente indirizzate al costruito storico e al paesaggio, con riferimento a ordini di grandezza e scale di restituzione conseguenti. Risulta, invece, ancora poco affrontata la codifica e la formalizzazione di pratiche di rilievo per oggetti di piccole dimensioni, con accuratezza e precisione necessariamente più elevate, anche nel caso di ricorso a strumentazioni "image-camera" piuttosto che range-camera.

A tale proposito si presenta il caso studio relativo alla documentazione fotogrammetrica di una lamina orfica, particolarmente cogente al fine di affrontare due problematiche al contempo: quella dimensionale e quella delle superfici riflettenti (come l'oro, con cui l'oggetto è realizzato).

L'acquisizione, pertanto, è stata condotta secondo due paradigmi: mediante un sistema originario "macro", con ottiche fotografiche dedicate, in condizioni di luce artificiale ottenute sviluppando una specifica tecnica di illuminazione (la Ring Reflection Lighting - RRL), e attraverso un sistema 'micro', un microscopio digitale con luce polarizzata.

I risultati delle sperimentazioni sono qui confrontati al fine di annodare le tessere di questa misterica laminetta nell'ambito del Progetto Visualizing Innovative and Social Artworks della Regione Calabria.
\end{abstract}

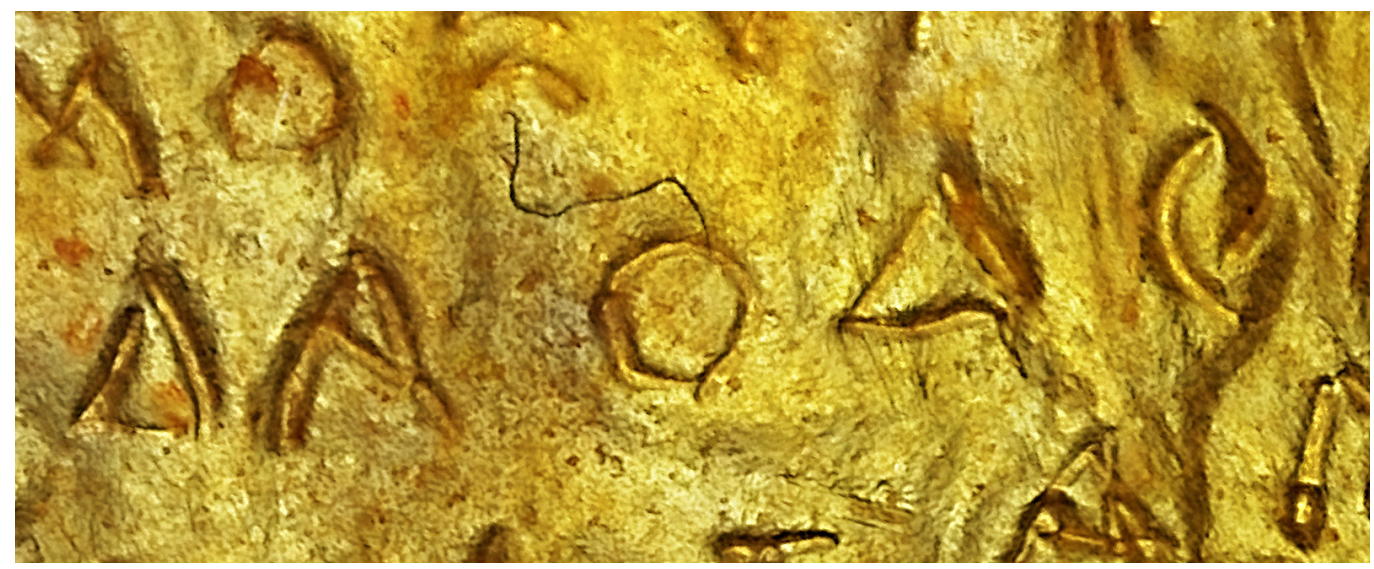




\section{Introduzione}

II contributo si concentra sulle sfide poste alla fotogrammetria allorquando si approcci il rilievo di oggetti di dimensioni ridotte e in condizioni di luce difficili. Nel dettaglio, infatti, ci si propone di illustrare le soluzioni procedurali individuate per portare a compimento il rilievo fotogrammetrico di una laminetta orfica risalente al V-IV secolo a.C., rinvenuta [I] presso Hipponion [Pugliese Carratelli 200 I, p. 17] e oggi conservata presso il Museo Nazionale di Vibo Valentia (fig. I).

II rilievo di questo reperto, per poter giungere a una qualità descrittiva adeguata, ha imposto la soluzione di tre condizioni intrinseche ed estrinseche: le sue piccole dimensioni (larghezza massima di $59 \mathrm{~mm}$, minima di $49 \mathrm{~mm}$ e altezza pari a $32 \mathrm{~mm}$ ); la presenza di un testo realizzato con microincisioni che presentano depressioni che difficilmente giungono ai 2,0 mm; il materiale altamente riflettente (oro) di cui è costituito. Queste peculiarità hanno reso evidenti numerose criticità nella fase di presa, che si sono tradotte nella progettazione e assemblaggio di un originario 'set di acquisizione', abbinato a una fotocamera full frame con ottiche macro. Qualità, operabilità e costi di questo metodo sono state poi raffrontate con i dati ricavati da un microscopio digitale portatile. Quanto osservato in questa esperienza risulta valevole al fine di poter avanzare un protocollo per la documentazione di piccoli manufatti, con dimensioni lineari fino a circa $5 \mathrm{~cm}$, ovvero efficace per una gamma di materiali frequentemente presenti nelle collezioni museali.

Fig. I. La lamina orfica di Hipponion (odierna Vibo Valentia) rinvenuta nel 1969 in una necropoli situata nella parte bassa della città.

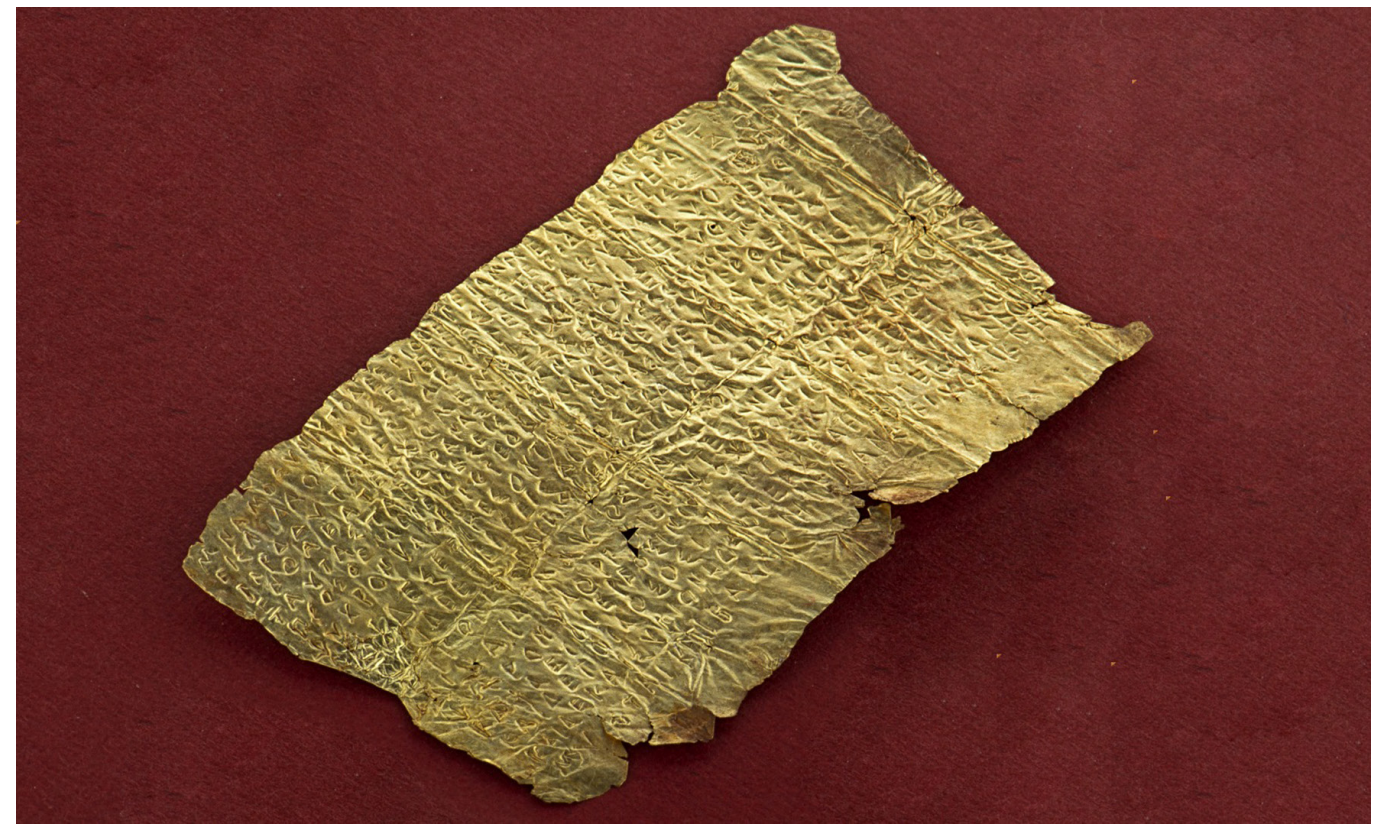

\section{Premessa metodologica}

La modellazione 3D basata sulla fotogrammetria digitale è diventata uno degli strumenti più diffusi per rispondere alle esigenze correlazionate al Cultural Heritage, per i requisiti prestazionali e di analisi [García Fernández, Álvaro Tordesillas, Barba 2015 p. 25-29], rappresentando uno straordinario mezzo per garantire la conservazione del bene, massimizzandone al contempo la fruizione. Ciò è di grande attualità, vista, inoltre, la grande mole di testimonianze giacenti nei magazzini dei nostri musei, il cui accesso è spesso limitato anche per sole finalità di studio.

In questo contesto operativo la fotogrammetria costituisce un metodo di rilevamento che presenta il vantaggio principale di essere - generalmente - a basso costo, portatile, flessibile 
e in grado di fornire allo stesso tempo geometrie e texture altamente dettagliate [Nicolae et al. 20 I 4, p. 45 I , 456]. Per di più, l'approccio fotogrammetrico comporta - intrinsecamente - la precisione metrica nel posizionamento dei singoli pixel sulla superficie e ciò è a garanzia di una fusione metricamente corretta, tra texture altamente dettagliate e mappe di normali [Bolognesi, Fiorillo, 2018, p.337-342].

Queste affermazioni trovano un riscontro operativo, però, solo se corroborate da una solida conoscenza - spesso troppo semplicistica - sia della fotografia e del processo fotogrammetrico [Marshall et al. 2019 p. 75I-757], sia della geometria proiettiva: condizione necessaria e sufficiente, insieme a una minuziosa oggettiva pianificazione della campagna di acquisizione, per un reale e completo controllo delle condizioni al contorno del soggetto (spazi limitati, possibilità o impossibilità di spostare l'oggetto, condizioni di illuminazione ecc.).

\section{Strumenti e procedure di acquisizione}

\section{Scanner VIVID 910}

Un primo tentativo di acquisire l'oggetto è stato condotto con lo scanner VIVID 9I0, un sistema senza contatto della KONIKA-MINOLTA basato sulla triangolazione laser (fig. 2). Lo strumento, dotato di tre ottiche intercambiabili a seconda delle esigenze, è stato utilizzato con l'obiettivo 'telescopic', specifico per superfici dettagliate di piccole dimensioni, a cui corrisponde, con una distanza di misurazione di $600 \mathrm{~mm}$, una superficie di cattura pari a $367 \times 275 \mathrm{~mm}$. La qualità di scansione è stata impostata su "fine", per un livello nominale di risoluzione dell'ordine del decimo di millimetro.

A causa dell'elevata riflettanza superficiale dell'oro, che determina reazioni molto differenti alle sollecitazioni esterne, la risposta della superficie da documentare è difficilmente prevedibile: di conseguenza, sono stati riscontrati e osservati diversi problemi, originati principalmente dalle caratteristiche superficiali oltre che dalla combinazione di angolo di scansione e condizione macroscopica locale (causa di perdite del segnale di ritorno).

I risultati, insoddisfacenti, soprattutto per quanto riguarda il dettaglio delle microincisioni, hanno condotto alla ricerca di soluzioni più performanti.

Fotocamera full-frame e ottiche macro

La qualità del dato fotografico, come noto, dipenderà principalmente dalla luce esterna, dalle caratteristiche ottiche della fotocamera e dalla risoluzione strumentale, intesa nella sua duplice declinazione di risoluzione geometrica (GSD) e radiometrica [Russo 2019, p. 997-1002].

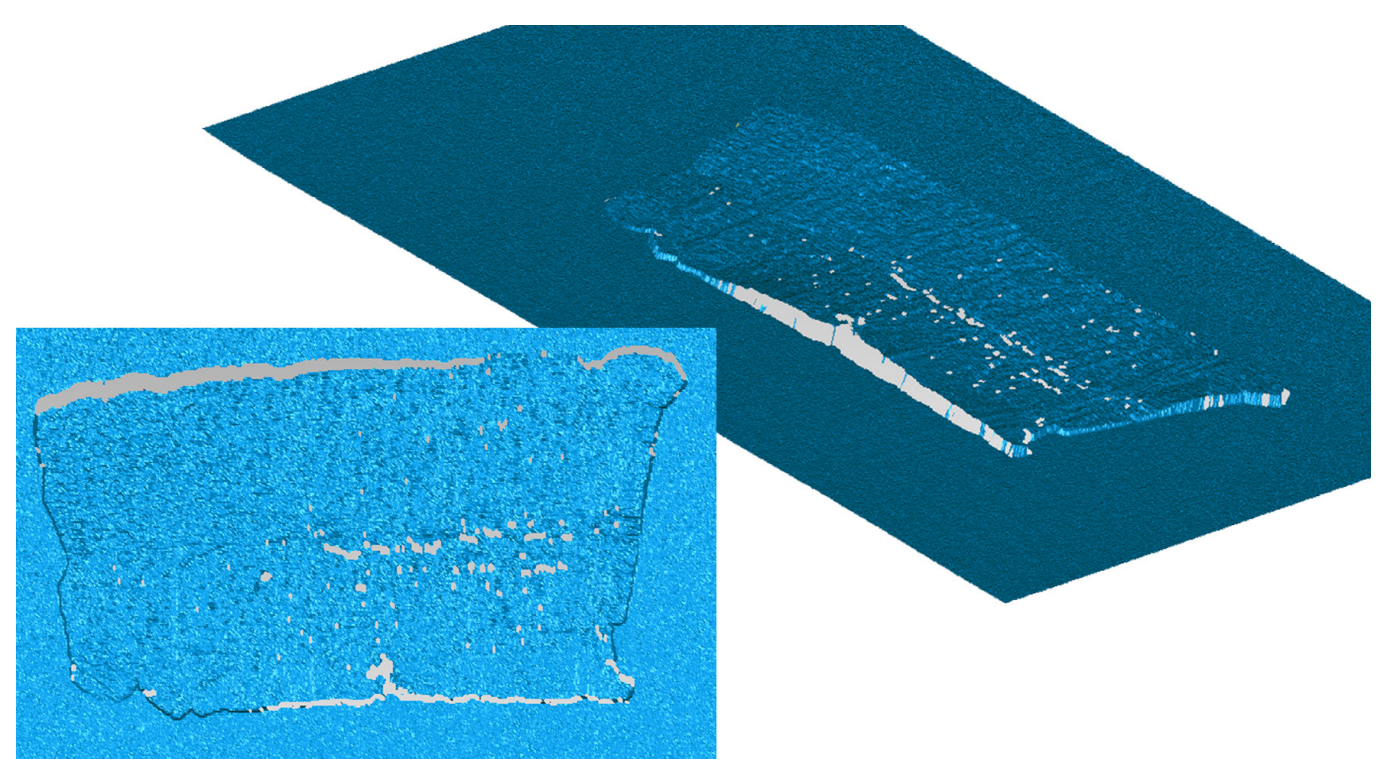


Al fine di massimizzare la qualità del dato, si considera in prima istanza la scelta del sistema ottico: proprio come accade per l'occhio umano, anche gli obiettivi fotografici sono in grado di effettuare il focamento solo fino a una certa distanza dal soggetto. Dal momento che nella maggior parte degli obiettivi tale distanza è progettata per rientrare nell'ordine del metro, effettuare riprese molto ravvicinate può risultare problematico. Gli obiettivi che più si prestano al caso sono denominati macro e, oltre a consentire una messa a fuoco ravicinata, rappresentano anche, fra le tipologie di obiettivi, quelli otticamente meno distorti. Nel nostro caso è stato utilizzato un AF-S VR Micro-Nikkor 105 mm f/2.8G IF-ED, che consente una distanza minima di messa a fuoco pari a 0,314 m.

Malgrado i pregi di queste ottiche, quali la ripresa ravvicinata e l'elevatissimo livello di dettaglio raggiungibile - di gran lunga superiore a quello delle ottiche comuni - le macro, sinora, sono state comunemente considerate adatte solo a un ristretto campo di applicazioni fotogrammetriche a causa della ridotta profondità di campo che le caratterizza. Nonostante in fotogrammetria l'accatastamento di immagini multi-focalizzate possa essere un'efficace contromisura a una profondità di campo limitata, ciò, tuttavia, aumenterebbe notevolmente i dati di input e appesantirebbe inutilmente la pipeline di elaborazione e il calcolo computazionale [Sapirstein 20 I8, p. 33-45].

Quindi, si è scelto di gestire il problema dello sfocato partendo dal concetto secondo il quale la profondità di campo aumenta al diminuire dell'apertura del diaframma [Greenleaf Allen 1950, p. 25-27], con conseguente riduzione della quantità di luce passante e aumento relativo [2] dei tempi di esposizione.

In base alla fotocamera digitale utilizzata - Nikon D800E, con sensore CMOS da 35,9×24,0 $\mathrm{mm}$ e 36,3 MP, per la quale si assume un cerchio di confusione pari a 0,0 I $46 \mathrm{~mm}$ - si è imposto una distanza tra centro ottico dell'obiettivo e soggetto pari a $70 \mathrm{~cm}$, impiegando una apertura di diaframma pari a f/32. Così facendo si è garantita una profondità di campo di $35,4 \mathrm{~mm}$, per la quale si accetta un lieve calo di nitidezza ai limiti dell'inquadratura, provocato da alcune riprese non ortogonali al piano dell'oggetto, però necessarie ai fini fotogrammetrici. A margine di sicurezza, in fase di campagna, ci si è allontanati dalla laminetta di ulteriori $10 \mathrm{~cm}$, mantenendo un'apertura di diaframma pari a f/32 ed estendendo la profondità di campo [3] a 49,8 $\mathrm{mm}$.

Ring Reflection Lighting

È stato poi oggetto di considerazioni e scelte il sistema di presa e le particolari condizioni di illuminazione da garantire: trattandosi di un piccolo oggetto, abbiamo immaginato, per una più efficiente acquisizione, di collocarlo su una base rotante con condizioni di luce diffusa per neutralizzare i coni d'ombra - senza variazioni di intensità di ombre, luci e colori -, così da avere una perfetta illuminazione per ogni angolo di rotazione della base. L'obiettivo è stato quello di avere un'illuminazione vantaggiosa in termini di ripresa fotografica e che, al contempo, non compromettesse - anzi eventualmente migliorasse - la ricostruzione della geometria. A questo fine è stato necessario impiegare un tipo di luce che non incidesse direttamente sull'oggetto; a tale scopo la laminetta, assieme alla base rotante con fondo opaco, è stata posta su un pannello orizzontale costituito da un materiale diffusore, un pannello in plexiglas, retroilluminato da una torcia flash.

La luce proveniente dal basso è riflessa sull'oggetto grazie all'impiego di un cilindro in forex bianco, concentrico alla base rotante, ma di diametro maggiore, cosicché la differenza fra i diametri determini un anello di luce che rimbalza sulla superficie cilindrica - che funge, quindi, da pannello riflettente (fig. 3) - illuminando uniformemente l'oggetto.

Si osservi che se fossimo stati in condizioni di luce continua, con il diaframma piuttosto chiuso, avremmo registrato tempi di esposizione lungo almeno una decina di secondi. Ciò non costituisce un problema disponendo di un treppiede, ma alcuni fattori sono comunque da non sottovalutare: ad esempio il rumore di crominanza [4], che crescerà all'aumentare del tempo di esposizione, così come l'incidenza della luce della scena sull'oggetto. Tale ultimo fattore aumenta anch'esso all'incremento del tempo di esposizione, nonostante siano imposte condizioni di luce artificiale; pertanto, l'unica chance per evitare tale influenza luminosa è stata impostare un tempo di esposizione breve e, avendo un diaframma chiuso, bilanciarlo 
Fig. 3. Schema del sistema RRL assemblato per un'illuminazione di presa diffusa. Partendo dal basso: beauty dish dal basso: beauty dish (sorgente luminosa), diffonden diffondente); tavola rotante, clindro in forex bianco (pannello riflettente)

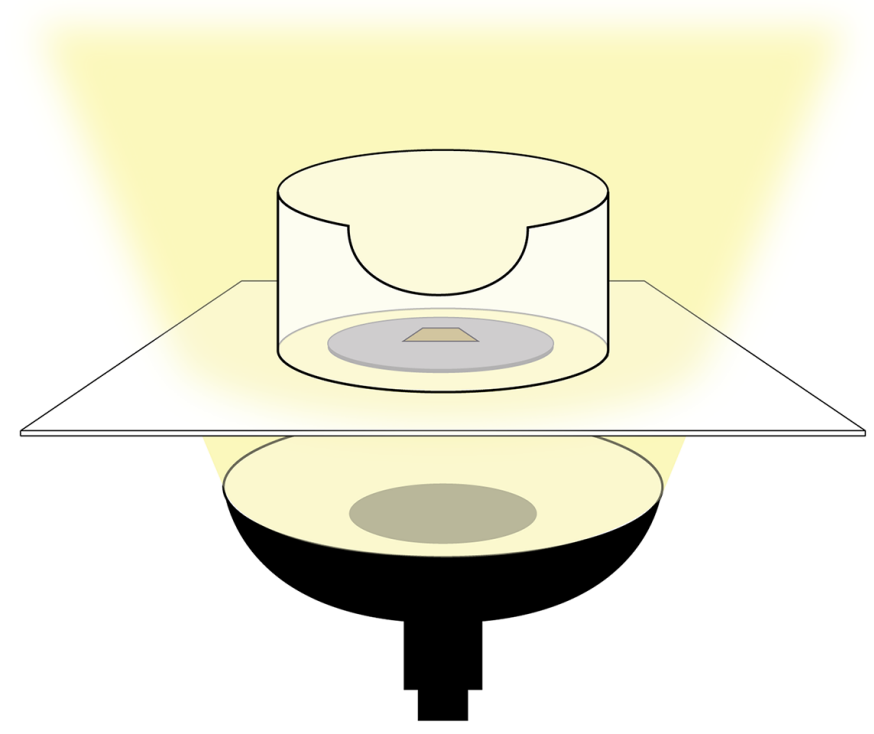

con una potenza luminosa tale da garantire la corretta esposizione (programmabile sulla centralina flash).

Con questo originale sistema, a cui è stato dato il nome di Ring Reflection Lighting - RRL e dal costo di assemblaggio approssimativo di 5.000 euro, sono state acquisite 72 immagini (una ogni $10^{\circ}$ di rotazione della base, con due differenti angoli di inclinazione, $30^{\circ}$ e $45^{\circ}$ ). Il tempo necessario tra allestimento del sistema e acquisizione (fig. 4) è stato di circa 40 minuti.

Dino-Lite AM4 I I 3 T

La necessità di verificare i risultati, la possibilità di implementare una strumentazione più economica e speditiva rispetto a quella illustrata precedentemente, così come testare l'accuratezza della stessa, ci ha spinto a confrontare gli output ottenuti con ottiche fotografiche macro a quelli ottenibili con un sistema di tipo microscopico digitale portatile, il Dino-Lite AM4I I 3T. Questo strumento rientra nell'ampia gamma di microscopi dotati di fotocamera digitale impiegati negli ultimi anni principalmente per applicazioni mediche e industriali, ma che possono svelare un'efficacia anche nel campo dei beni culturali [Esmaeili, Ebadi 20 I7, p. 65-67]. Infatti, la loro leggerezza, la facilità d'uso e di presa, consentono di ottenere immagini con sovrapposizioni adeguate; di contro, l'angolo di campo invariabilmente stretto e la limitatissima profondità di campo possono rappresentare evidenti restrizioni.

II particolare strumento impiegato è caratterizzato da un sensore fotografico da I,3 MP, luce polarizzata, fattore di magnificazione fino a 200x ed è capace di apprezzare, in ambiente software proprietario, delle misure di distanze lineari; il relativo costo è dell'ordine dei 500 euro. Con il microscopio collegato a una workstation portatile, operando con la procedura dedicata di DinoCapture 2.0, sono state acquisite 490 immagini alla massima risoluzione (3,75 MB ciascuna), a una distanza operativa media di circa $2 \mathrm{~cm}$, con fattore di ingrandimento 30x. Le immagini sono state acquisite secondo il paradigma fotogrammetrico multi-immagine, garantendo, dunque, una sovrapposizione media tra i fotogrammi di circa il 70\%, muovendo manualmente il supporto della tavoletta con il microscopio assicurato su stativo (fig. 5). Il procedimento ha richiesto, includendo le operazioni di calibrazione metrica per il fattore di magnificazione scelto, circa 50 minuti. La necessità di acquisire un elevato numero di immagini per la documentazione della laminetta è derivata dal fatto che questo microscopio digitale è caratterizzato, alla distanza operativa scelta per l'acquisizione, da un FOV di I3,0×10,4 mm e da un DOF di I,9 mm. 
Fig. 4. Acquisizione della lamina con strumentazione 'macro'; in secondo piano l'anello di illuminazione RRL.

Fig. 5. Acquisizione della lamina con Dino-Lite AM4II3T.
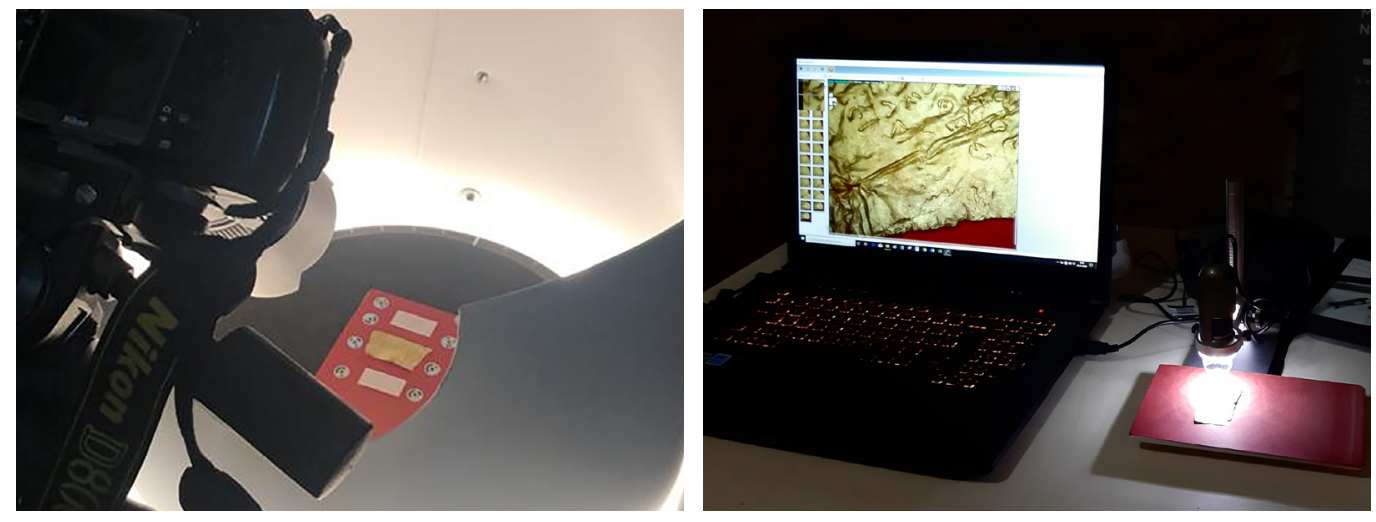

\section{II processo fotogrammetrico}

I due set di immagini sono stati processati in un software di tipo SFM (Structure from motion): RealityCapture. Entrambe le elaborazioni sono stata portate a termine con una workstation grafica dotata di CPU Intel 19 9900k, GPU RTX2080ti, 64GB RAM, con i progetti e i relativi file temporanei allocati su SSD M.2. In RealityCapture il data processing è articolato in tre step: Align Images (Max feature per image 70.000); Reconstruction (High detail); Texture. Per i dati acquisiti con sistema "macro", il tempo impiegato per processare I,I2 GB di immagini è stato di circa 50 minuti; in precedenza l'esposizione delle immagini era stata ottimizzata in Camera Raw, secondo un pre-processo che ha richiesto altri 15 minuti circa. Nel caso dei dati acquisiti con il sistema 'micro', il tempo impiegato per processare I,8 GB di immagini è stato di circa 60 minuti.

Alla fine delle elaborazioni sono state esportate, per entrambi gli approcci, un modello mesh con texture, per analisi di tipo quantitative e una prima ortofoto per analisi qualitative (la generazione e l'esportazione dell'ortofoto ha richiesto, in entrambi i casi, circa 10 minuti). La mesh proveniente dal sistema di presa 'macro' è composta da 6,7 milioni di poligoni, mentre quella realizzata con il Dino-Lite da 19,8 milioni di poligoni (fig. 6). Per quanto riguarda le ortofoto, dai dati ottenuti con la strumentazione fotografica è stata generata un'ortofoto (fig. 7) delle dimensioni di $8466 \times 604 \mathrm{I} \mathrm{px}\left(1,5 \cdot 10^{-3} \mathrm{~mm} / \mathrm{px}\right)$, mentre dai dati ottenuti con la strumentazione microscopica, l'ortofoto generata (fig. 8) è risultata di 24808×405/8 px $\left(7,8 \cdot 10^{-5} \mathrm{~mm} / \mathrm{px}\right)$.

\section{Validazione dei dati}

Preparazione dei dataset

I due modelli, utilizzando l'applicativo open source CloudCompare 2. I0.2, sono stati portati in unità reali $(\mathrm{mm})$ attraverso una scalatura diretta. Questa operazione è stata effettuata, nel caso del sistema 'macro', posizionando due scale bars nella scena (una per scalare, l'altra per verificare l'accuratezza dell'operazione). Nel secondo, considerando il FOV estremamente modesto del Dino-Lite, che avrebbe imposto l'acquisizione di alcune centinaia di immagini ulteriori per poter includere dei riferimenti nella scena, è stata realizzata adoperando il richiamato strumento di misura presente in DinoCapture 2.0. Questo strumento, dopo aver generato un profilo di calibrazione per il fattore di ingrandimento alla distanza prescelta, permette di prendere misure piane sufficientemente dettagliate direttamente dalle singole prese. Quindi, dopo aver ripristinato il corretto fattore di scala per le due superfici restituite, al fine di poterne confrontare la qualità descrittiva, sono state allineate prima collimando punti omologhi e, successivamente - al fine di raffinare la roto-traslazione rigida così ottenuta - è stato eseguito un allineamento automatico per mezzo di un algoritmo ICP (Iterative Closest Point). 
Fig. 6. Nuvola fotogrammetrica e mesh risultante

(in rosso) gen

sistema 'micro'.

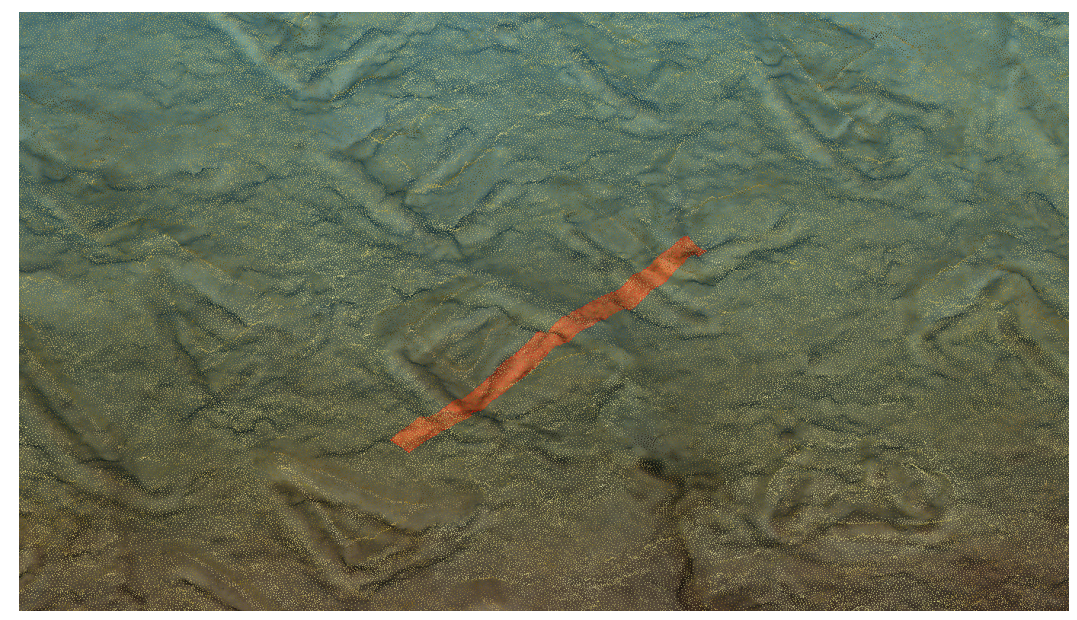

Fig. 7. Ortofoto generate con il dataset prove-

niente dall'acquisizione

fotografica.

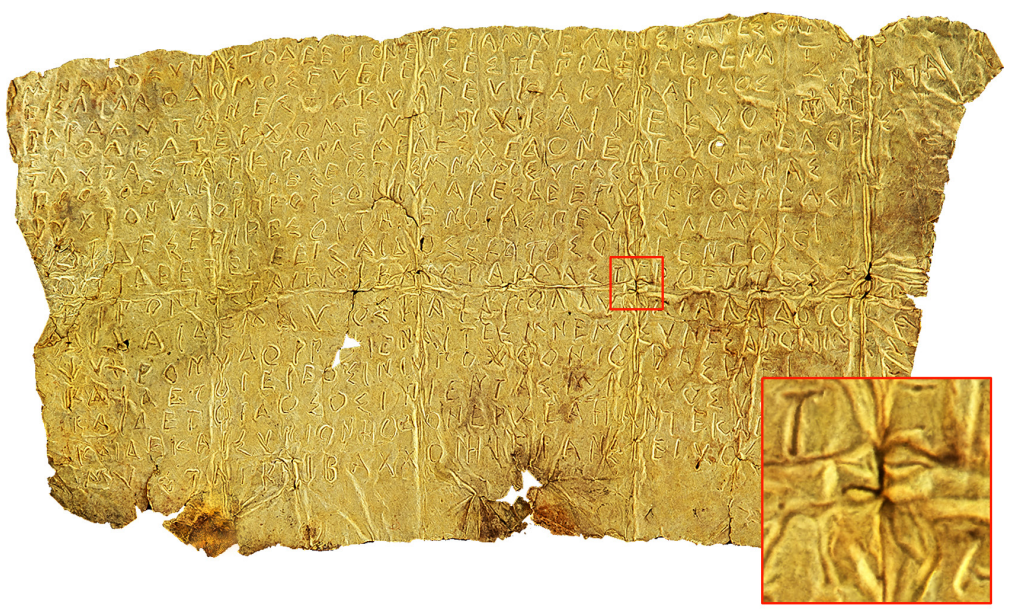

Fig. 8. Ortofoto generate con il dataset proveniente dall'acquisizione microscopica.

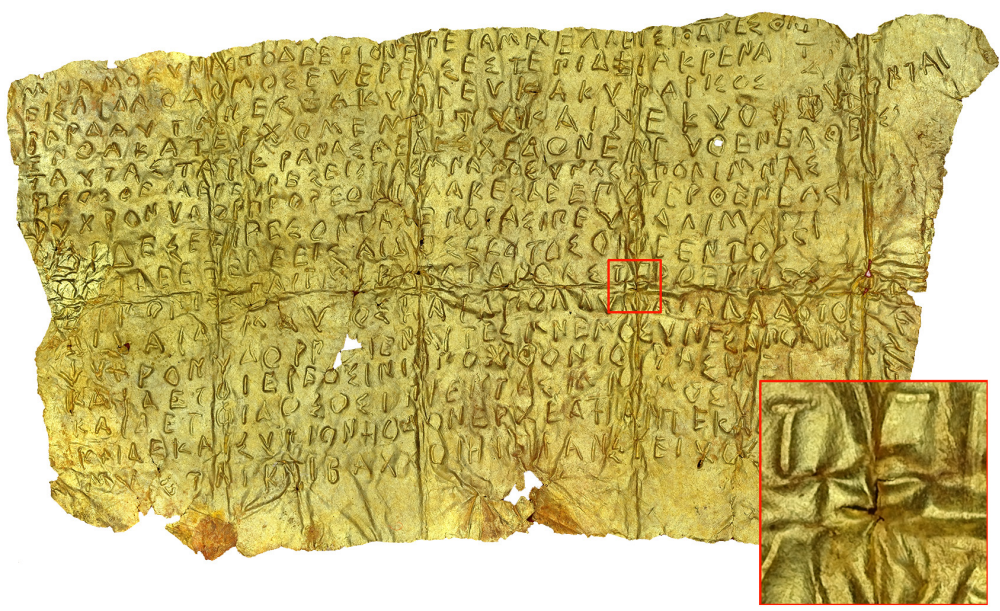


Analisi relative dei dataset

La fase di valutazione della qualità descrittiva delle superfici è stata effettuata sempre nell'ambiente open source di CloudCompare, mediante lo strumento C2C che permette di valutare, per ogni punto della cloud o triangolo della mesh che si sta confrontando, quello più vicino nell'entità di rifermento, definendo quindi un valore di scostamento del primo rispetto al secondo. Quindi, impostando come riferimento la cloud generata con il Dino-Lite, è stata confrontata quella ottenuta dal sistema 'macro', evidenziando, sinteticamente e in falso colore, gli scostamenti delle superfici, compresi tra $-5 \mathrm{~mm}$ e $0 \mathrm{~mm}$ (fig. 9): i calcoli effettuati mostrano, infatti, deviazioni medie non superiori ai $2 \mathrm{~mm}$.

Si osservi, inoltre, che le deviazioni maggiori ( $>$ di $5 \mathrm{~mm}$ ) sono presenti soprattutto sulle parti marginali della scena. Questa discrepanza, in particolare, è dovuta dalla distanza di queste stesse zone dal punto di messa a fuoco, a seguito dell'impiego di una tavola rotante per le acquisizioni delle prese fotografiche con il sistema "macro". Infatti, pur permettendo una rapida successione di scatti a una distanza fissa dal soggetto, regolando solo l'altezza della fotocamera ed evitando il continuo riposizionamento del treppiede, ciò costringe a inquadrare l'intero oggetto in ogni singola presa, riducendo significativamente il dettaglio del dato. La differenza fra i due modelli nelle zone marginali è inoltre ancor più accentuata dallo schema a prese ortogonali estremamente ravvicinate seguito col Dino-Lite, i cui dati input non risultano, pertanto, affetti da alcuna significativa perdita di dettaglio.

Fig. 9. Comparazione cloud to cloud in
ambiente Cloud Compare ambiente Cloud Compar per la valutazione le nuvole ottenute con due differenti tecniche $d$ acquisizione.

Fig. 10. Confronto di una sezione di nuvola di punt: in color per vertex il dato cor sistema 'micro', in bianco quello 'micro', in bianco quello generato col sistema 'macro'.
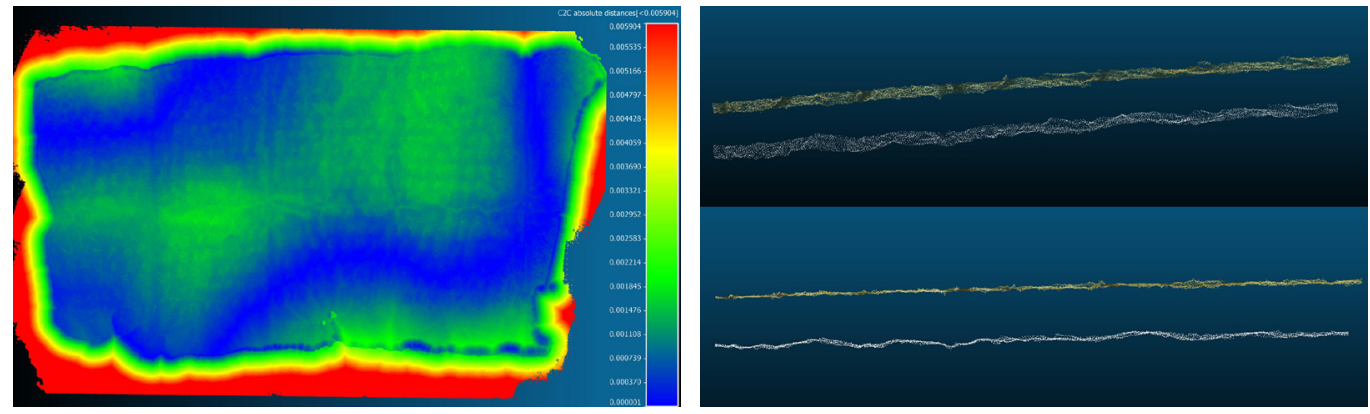

\section{Prime conclusioni}

Ingombro dell'attrezzatura ed economicità, con un prezzo inferiore del $90 \%$ e qualità generale del dato - soprattutto di quello bidimensionale -, fanno propendere per una validità dell'applicazione del Dino-Lite AM4I I 3T nel campo dei beni culturali, come soluzione innovativa da adottare prevalentemente per piccoli bassorilievi (fig. I I). Per quanto riguarda la descrizione tridimensionale della superficie si riscontra una differenza modesta tra i due sistemi, con una qualità moderatamente migliore del dato del Dino-Lite AM4 I I 3 T rispetto al sistema 'macro', relativamente alla descrizione delle microincisioni; diversamente, la forma generale della laminetta appare migliore nel rilievo ottenuto con quest'ultimo sistema (fig. I0). II risultato può essere migliorato attraverso l'integrazione delle due tecniche, la qualcosa richiederà lunghe e macchinose attività di registrazione delle posizioni delle camere, ciò al fine di garantire la bontà della geometria ottenuta con il sistema "macro" e i dettagli e i costi del "micro".

Ulteriori sperimentazioni verranno condotte mettendo a confronto il sistema microscopico con attrezzature a luce strutturata e tecniche fotografiche finalizzate a ottenere maggiore dettaglio utilizzando dispositivi ottici di prolunga [5] (con una riduzione della distanza tra sistema ottico e oggetto). Così operando sarà possibile focalizzare più da vicino il soggetto, avere un grado di ingrandimento maggiore, incrementare il dettaglio dell'acquisizione e annodare le tessere per rileggere la storia misterica di questa laminetta nell'ambito del Progetto Visualizing Innovative and Social Artworks della Regione Calabria. 
Fig. I I. Sintesi delle principali caratteristiche dei due sistemi di acquisizione implementati.

\begin{tabular}{|c|c|c|c|c|c|c|c|c|c|c|c|c|}
\hline & EQUIPMENT & PRICE & SUPPLY & $\begin{array}{l}\text { ACQUISITION } \\
\text { RUNNING TIME }\end{array}$ & $\begin{array}{l}\text { NUMBER OF } \\
\text { ACQUISITIONS }\end{array}$ & $\begin{array}{l}\text { SIZE } \\
\text { OF EACH }\end{array}$ & $\begin{array}{l}\text { TOTAL } \\
\text { SIZE }\end{array}$ & MF & DOF & PIXEL SIZE & $\begin{array}{l}\text { ORTOPHOTO } \\
\text { DIMENSIONS }\end{array}$ & $\begin{array}{l}\text { NUMBER OF } \\
\text { POLYGONS }\end{array}$ \\
\hline $\begin{array}{l}0 \\
\frac{\pi}{0} \\
\frac{0}{E}\end{array}$ & $\begin{array}{l}\text { Dino-Lite } \\
\text { AM4113T }\end{array}$ & $€ 500$ & $\begin{array}{l}\text { battery } \\
\text { powered }\end{array}$ & $\begin{array}{l}50 \text { min } \\
\text { no set up } \\
\text { needed }\end{array}$ & 490 & 3,8 MB & $1,8 \mathrm{~GB}$ & $30 x$ & $1,9 \mathrm{~mm}$ & $1,5 \cdot 10^{-3} \mathrm{~mm} / \mathrm{px}$ & $24.808 \times 40.518 \mathrm{px}$ & $19,8 \times 10^{6}$ \\
\hline $\begin{array}{l}\frac{0}{0} \\
\frac{0}{0} \\
\frac{\pi}{2}\end{array}$ & $\begin{array}{l}\text { Nikon D800E + } \\
\text { AF-S VR Micro-Nikkor } \\
105 \mathrm{~mm} \text { f/2.8G IF-ED + } \\
\text { Bowens Flash Quad + } \\
\text { RRL Cylinder }\end{array}$ & $€ 5.000$ & $\begin{array}{l}\text { power } \\
\text { supply }\end{array}$ & $\begin{array}{l}40 \mathrm{~min} \\
\text { set up } \\
\text { included }\end{array}$ & 72 & $72,8 \mathrm{MB}$ & $2,5 \mathrm{~GB}$ & $0,15 x$ & $49,8 \mathrm{~mm}$ & $7,8 \cdot 10^{-5} \mathrm{~mm} / \mathrm{px}$ & $8.466 \times 6.041 p x$ & $6,7 \times 10^{6}$ \\
\hline
\end{tabular}

Note

[I] La lamina orfica rappresenta un'importante testimonianza del culto delle religioni misteriche in Calabria (forse già in uso prima dell'arrivo dei greci). Deposta piegata in 4 parti, sul petto del defunto - che nel caso era una fanciulla di nobile famiglia - aveva lo scopo di accompagnare la sua anima e guidarla nel regno di Ade.

[2] L'aumento del tempo di esposizione è qui definito 'relativo' perché dipende, oltre che dall'apertura di diaframma, anche dalla quantità di energia radiante disponibile nell'unità di tempo, ovvero la potenza luminosa erogata dalla sorgente artificiale. Appare banale osservare che, a parità di diaframma e in condizioni di luce continua (come ad esempio in esterno), il tempo sarà univocamente determinato dal valore di tale quantità, fissa a priori; in condizioni di luce artificiale flash, invece, accade che il tempo possa essere determinato dall'operatore, impostando a monte la potenza del generatore.

[3] I valori esplicitati derivano dalla formalizzazione matematica del problema nel campo delle ipotesi semplificative introdotte dall'ottica geometrica.

[4] II rumore va inteso come un disturbo che si sovrappone a un segnale 'perfetto', costituito da due componenti sovrapposte: il rumore di luminanza e il rumore di crominanza. II primo è proporzionale al valore degli ISO, il secondo è in funzione del tempo di esposizione.

[5] Dispositivi di estensione del sistema ottico da posizionare tra l'obiettivo e il corpo della fotocamera: allontanando il sistema ottico dalla fotocamera, la sua componente frontale risulterà più vicina al soggetto garantendo, nonostante ciò, una messa a fuoco.

\section{Riferimenti bibliografici}

Bolognesi Cecilia Maria, Fiorillo Fausta (2018). Optimization of texture mapping process in the Reality-Based Modeling application. In Salerno Rossella (a cura di). Rappresentazione/Materiale/lmmateriale. $40^{\circ}$ Convegno internazionale dei Docenti delle Discipline della Rappresentazione. Milano 15- 17 Settembre 20 18. Roma: Gangemi Editore International, pp. 337-342.

Greenleaf Allen R. (1950). Photographic optics. New York: Macmillan.

García Fernández Jorge, Álvaro Tordesillas Antonio, Barba Salvatore (20I5). An approach to 3D digital modeling of surfaces with poor texture by range imaging techniques. 'Shape from stereo' vs. 'Shape from silhouette' in digitizing Jorge Oteiza's sculptures. In The International Archives of the Photogrammetry, Remote Sensing and Spatial Information Sciences, Volume XL-5/ W4, pp. 25-29. 3D Virtual Reconstruction and Visualization of Complex Architectures, 25-27 February, Avila, Spain.

Esmaeili Farid, Ebadi Hamid (2017). Handy microscopic close-range videogrammetry. In The International Archives of the Photogrammetry, Remote Sensing and Spatial Information Sciences, Volume XLII-4/W4, 2017, pp. 65-67. Tehran's Joint ISPRS Conferences of GI Research, SMPR and EOEC 2017, 7-10 October 2017.

Marshall Matt, Johnson Andrew, Summerskill Steve, Baird Quinner, Esteban Enrique (2019). Automating photogrammetry for the 3d digitisation of small artefact collections. In The International Archives of the Photogrammetry, Remote Sensing and Spatial Information Sciences, Volume XLII-2N I 5, 2019, pp. 75 I-757. 27th CIPA International Symposium "Documenting the past for a better future", I-5 September, Ávila, Spain.

Nicolae Corina, Nocerino Erica, Menna Fabio, Remondino Fabio (20 I4). Photogrammetry applied to problematic artefacts. In The International Archives of the Photogrammetry, Remote Sensing and Spatial Information Sciences, Volume XL-5, pp. 45I -456. In ISPRS Technical Commission V Symposium, 23-25 june, Riva del Garda, Italy.

Pugliese Carratelli Giovanni (200 I). Le lamine d'oro orfiche. Istruzioni per il viaggio oltremondano degli iniziati greci. Milano: Adelphi.

Russo Michele (2019). Fotomodellazione 2020: viaggio di sola andata? In Belardi Paolo (a cura di). Riflessioni, l'arte del disegno/ il disegno dell'arte. $41^{\circ}$ Convegno internazionale dei docenti delle discipline della Rappresentazione. Perugia I9-21 settembre 20 19. Roma: Gangemi Editore International, pp. 425-430.

Sapirstein Philip (2018). A high-precision photogrammetric recording system for small artifacts. In Journal of Cultural Heritage, 3I, pp. 33-45.

\section{Autori}

Sara Antinozzi, Università degli Studi di Salerno, santinozzi@unisa.it

Diego Ronchi, Spiron Heritage and Survey, Roma, diego.ronchi@spiron.it

Salvatore Barba, Università degli Studi di Salerno, sbarba@unisa

Per citare questo capitolo: Sara Antinozzi, Diego Ronchi, Salvatore Barba (2020). Macro e micro fotogrammetria per la virtualizzazione della laminetta orfica (V-IV a.C.) del Museo Nazionale di Vibo Valentia/Macro and Micro Photogrammetry for the Virtualization of the Orphic Foll (V-IV B.C) of National Museum of Vibo Valentia. In Arena A., Arena M., Brandolino R.G., Colistra D., Ginex G., Mediati D., Nucifora S., Raffa P. (a cura di). Connettere. Un disegno per annodare e tessere. Atti del $42^{\circ}$ Convegno Internazionale dei Docenti delle Discipline della Rappresentazione Connecting. Drawing for weaving relationships. Proceedings of the 42th International Conference of Representation Disciplines Teachers. Milano: FrancoAngeli, pp. 1538-1555. 


\title{
Macro and Micro Photogrammetry for the Virtualization of the Orphic Foil (V-IV B.C.) of National Museum of Vibo Valentia
}

\author{
Sara Antinozzi \\ Diego Ronchi \\ Salvatore Barba
}

Abstract

Discussing photogrammetric surveying methods does not in itself constitute an innovative activity. However, modern application deserve an innovative and critic approach not only by a technological evolution of acquisition tools, but also for the challenge posed to us detectors and most commonly to the theory of measurement. The new opportunities for describing and representing reality are most commonly redirected to the historical built and the landscape, referring to consequent orders of magnitude and restitution scales. The codification and formalization of surveying techniques for small objects with high precision and accuracy, also in the case of "image-camera" and "range-camera" tools. For this reason is presented the case study of the photogrammetric acquisition of an orphic gold tablet, particularly challenging in order to discuss two problems at the same time: the size and the reflective material (gold with which the object is made).

The acquisition is carried out with two different instrumentations: using a native 'macro' photographic system, with dedicated photographic lens, in artificial light conditions obtained by developing a specific lighting technique (Ring Reflection Lighting - RRL) and using a 'micro' system through digital microscope with polarized light.

The results are compared in order to generate weaving relationships between of this foil within the Visualizing Innovative and Social Artworks project of Calabria Region.

Keywords

small artefacts, reflective objects, Ring Reflection Lighting, Dino-Lite, Reality Capture.

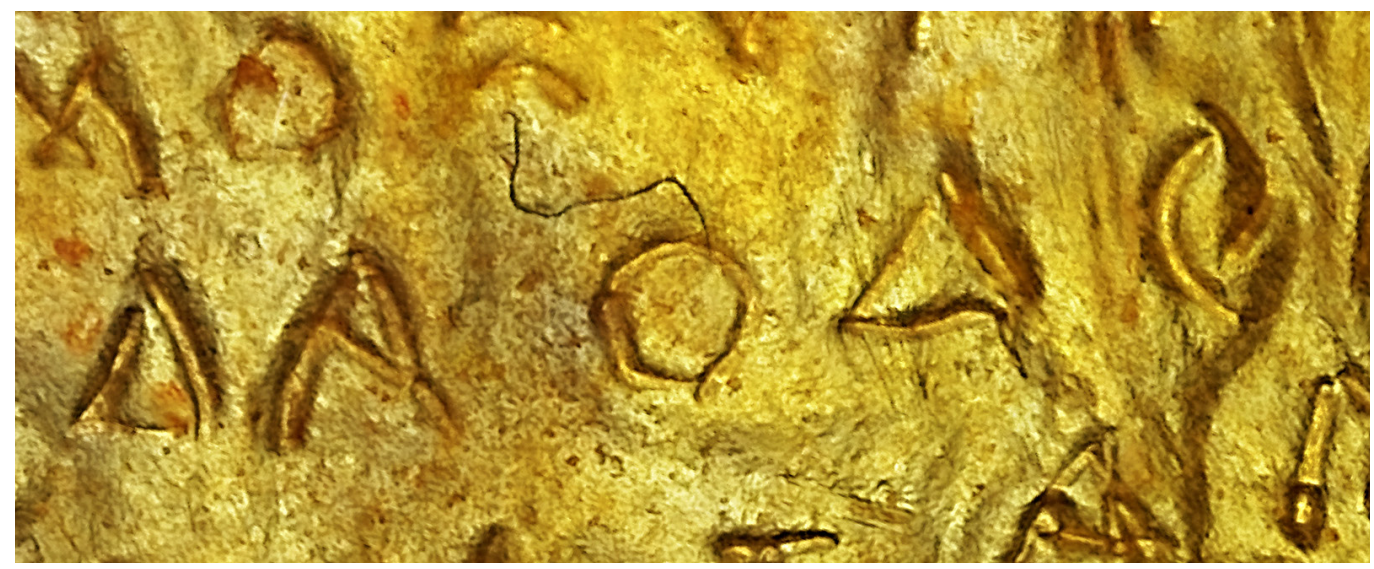




\section{Introduction}

This paper focuses on challenges posed by photogrammetry approaching the survey of small objects in difficult light conditions. In detail, it is proposed to illustrate the procedural solutions identified to carry out the photogrammetric survey of an orphic gold tablet dating V-IV century b.C., found [I] at Hipponion [Pugliese Carratelli 200 I, p. I 7] and now preserved in the National Museum of Vibo Valentia (fig. I).

The survey of this finding, in order to reach an adequate descriptive quality, required to solve three intrinsic and extrinsic conditions: its small size (maximum length: $59 \mathrm{~mm}$, minimum length: $49 \mathrm{~mm}$; height: $32 \mathrm{~mm}$ ); the presence of a text made with micro-engravings that have a maximum relief of $2,0 \mathrm{~mm}$; the highly reflective material (gold) of which it is made. These particular features of the object make evident some critical aspects of the acquisition phase, critical issues that, in the first instance, result in the design and in the construction of an "acquisition set" combined with a full frame camera and macro lens. The quality, speed and cost-effectiveness of the first method were then compared with the data obtained from a portable digital microscope. What has been found in this experience is valid in order to propose a new pipeline for the documentation of small artefacts, up to about $5 \mathrm{~cm}$, effective for a range of materials frequently present in museum collections.

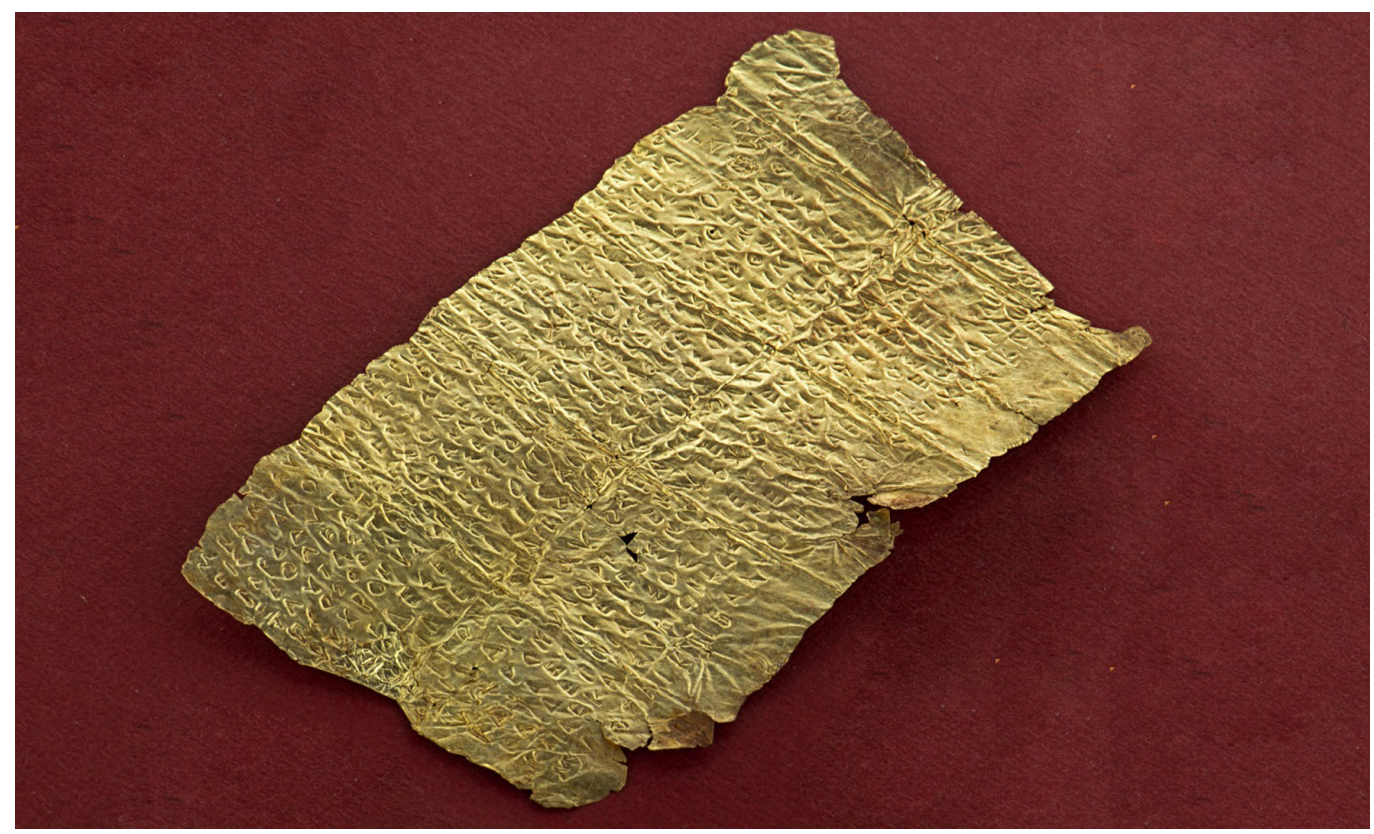

\section{Methodological premise}

The 3D digital modelling based on digital photogrammetry has become one of the most important tools in solving actual needs for heritage analyses, procedural modelling and accuracy requirement [García Fernández, Álvaro Tordesillas, Barba 20 I 5, p. 25-29] representing an extraordinary way to guarantee heritage conservation while maximizing their use. This is even more true for the large amount of finds stored in warehouses, whose use is often limited to study reasons only.

In this operational context, photogrammetry is a surveying method which has the major advantages of being -usually- low-cost, portable, flexible and able to deliver highly detailed geometries and textures at the same time [Nicolae et al. 20 I4, p. 45 I-456]. 
Furthermore the photogrammetric approach intrinsically involves the metric precision in the positioning of the single pixel on the surface and this guaranties a metrically correct fusion between high detailed texture and normal maps [Bolognesi, Fiorillo 2018, p.337342].

These assumptions are true, given a solid knowledge of both photography and photogrammetric process [Marshall and ot. 2019, p. 75I-757] and ensuring that the acquisition campaign is preceded by a meticulous work of planning and controllling the boundary conditions of the subject (limited spaces, possibility or impossibility of moving the object, lighting conditions).

\section{Equipment and acquisition procedures}

\section{VIVID 910 Scanner}

A first attempt to acquire the object has been made with the VIVID 910 3D Scanner, a non-contact system by KONIKA-MINOLTA based on laser triangulation (fig. 2).

The instrument, equipped with three interchangeable optics according to needs, was used with the "telescopic" lens, specific for small detailed surfaces, to which corresponds, with a measuring distance of $600 \mathrm{~mm}$, a catching surface at $367 \times 275 \mathrm{~mm}$.

The scan quality has been set to 'fine', for a nominal resolution level of the order of tenth of a millimetre.

Because of gold's high superficial reflectance, making it react so differently to external solicitations, the answer of this surface to the laser scanner beam is difficultly predictable: consequentially some problems are noticed and mainly originated by surface characteristics and by particular combination of scanning angle and local macroscopic condition (causing by a loose of back signal).

The unsatisfactory results, especially as regards the detail of the micro incisions, have led to the search for more performing solutions.

Full-frame camera and macro lens

In the acquisition phase, as is well known, the data quality mainly depends on the external light, on the optical characteristics of the camera and on the instrumental resolution, in its dual declination of geometrical (GSD) and radiometric resolution [Russo 2019, p. 997-1002].

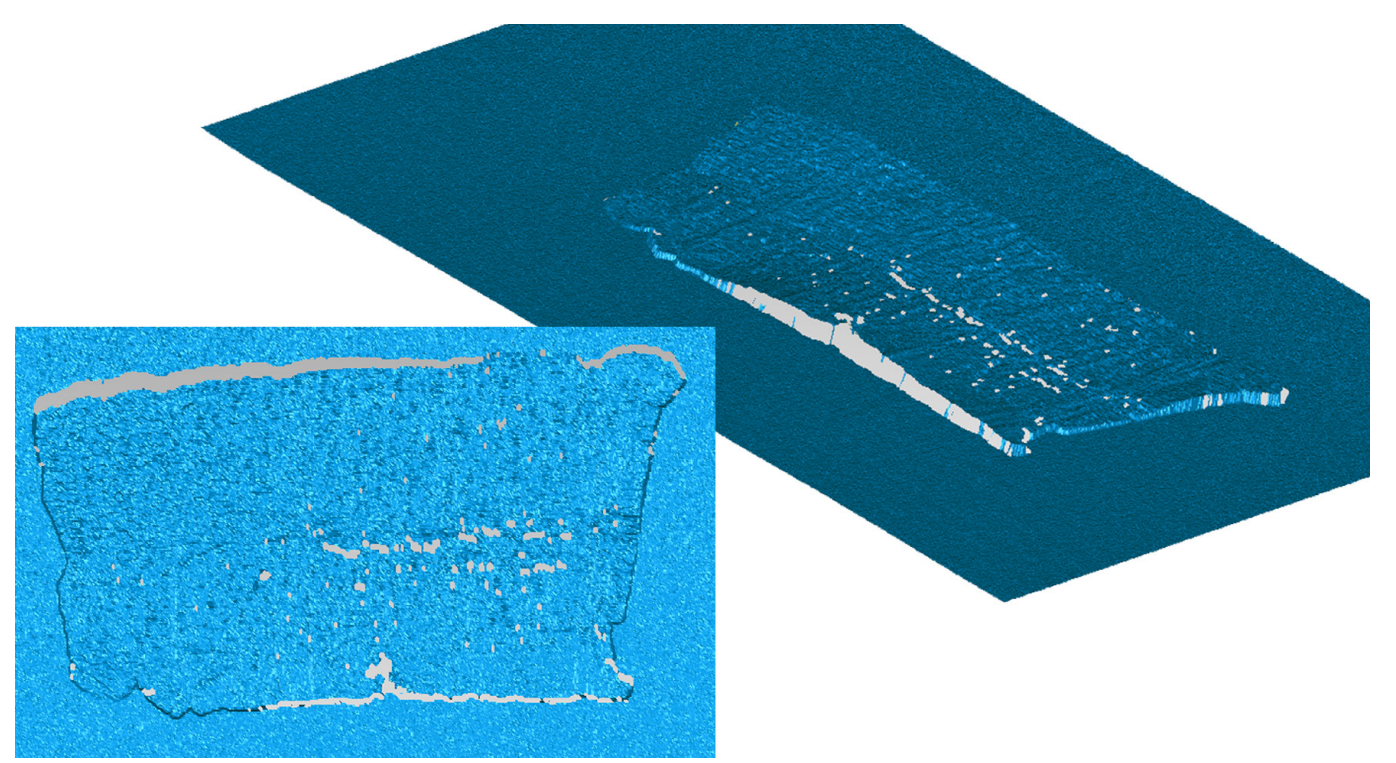


In order to maximize the data quality, first consider the choice of the optical system: as it happens for the human eye, even photographic lenses are capable of focusing only up to a certain distance from the subject. Since in most lenses this distance is designed to fall within the meter order, taking very close shots can be problematic. The most suitable objectives for this case are called macro and, as well as allowing the close focus, they also represent, among the types of objectives, those optically less affected by distortions. In our case, an AF-S VR Micro-Nikkor 105 mm f/2.8G IF-ED was used, which allows a minimum focusing distance of 0,314 m.

Despite the advantages related to macro optics, such as close-up shooting and the very high level of detail that can be reached, far superior to that of common optic systems, these are commonly considered suitable only for a narrow range of photogrammetric applications due to the very low depth of field. Even if multi-focused image stacking has been demonstrated as an effective countermeasure to restricted DOF with very small subjects, less than $2 \mathrm{~cm}$ across, however, it would significantly increase the number of photographs and unnecessarily burden the processing pipeline [Sapirstein 20।8, p. 33- 45]. Consequentially we have chosen to manage the problem of blur starting from the concept that the depth of field increases with the decrease of the aperture [Greenleaf Allen 1950, p. 25-27] with the consequent reduction of the amount of light passing through and the relative [2] increase in exposure times.

Depending on the digital camera used - Nikon D800E, characterized by a $35,9 \times 24,0 \mathrm{~mm}$ and 36,3 MP CMOS sensor, for which a confusion circle of 0,0 $446 \mathrm{~mm}$ is assumed - a distance is imposed between the optical centre of the lens and the object equal to 70 $\mathrm{cm}$, using an aperture of $\mathrm{f} / 32$. This way you can guarantee a depth of field of 35,4 mm, accepting a slight drop in sharpness caused by some shots not orthogonal to the plane of the object, but necessary for photogrammetric purposes. You put yourself in safety during the ongoing campaign - by moving away from the foil of $10 \mathrm{~cm}$ and maintaining an aperture of f/32, extending the depth [3] of field to $49,8 \mathrm{~mm}$.

Ring Reflection Lighting

Let's focus on the choice of the acquisition system and the lighting conditions: since it is a small and movable object, it has been identified, as the most efficient way to acquire it, that of placing it on a rotating base with diffused light conditions that neutralize the shadow cones - without variations in the intensity of shadows, lights and colours- so as to have perfect lighting for each rotation. The sense is to have a lighting that is advantageous in terms of photographic shooting and that at the same time does not compromise -indeed possibly improve- the reconstruction of the geometry. For this purpose, it was necessary to use a type of light that did not directly affect the object. Then, the gold tablet, together with the opaque bottom's rotating base, was placed on a horizontal panel consisting of a diffusing material, a plexiglass panel backlit by a flashlight.

The light coming from below is reflected on the object thanks to the use of a white forex cylinder, concentric to the rotating base, but built with a larger diameter, so that the difference between the diameters determines a ring of light that bounces on the cylindrical surface -acting then as a reflective panel (fig. 3)- uniformly illuminating the object.

Note that the diaphragm is rather closed and if we were in continuous light conditions this would entail an exposure time of ten seconds at least. This is not a problem if you have a tripod, but some factors should not be underestimated such as the chrominance noise [4], which increases as the exposure time increases and the incidence of the light from the scene on the object. The last factor grows with increasing exposure time, despite artificial light conditions imposed and the only chance to avoid this light influence is for the exposure to be short and, having a closed diaphragm, that it is balanced by a light power such as to guarantee the correct exposure (programmable on the flash unit).

Using this original system, named Ring Reflection Lighting - RRL, of the approximate assembly cost of $€ 5.000,72$ images were acquired (approximately one every 10 degrees base rotation with two different angles of tilting, $30^{\circ}$ and $45^{\circ}$ ). The time required between setting up the system and acquiring (fig. 4) is approximately 40 minutes. 
Fig. 3. Scheme of the assembled RRL system for diffused acquisition lighting. Starting from the bottom: beauty dish (light source); plexiglass top (diffusing base):rotating table; white forex cyling

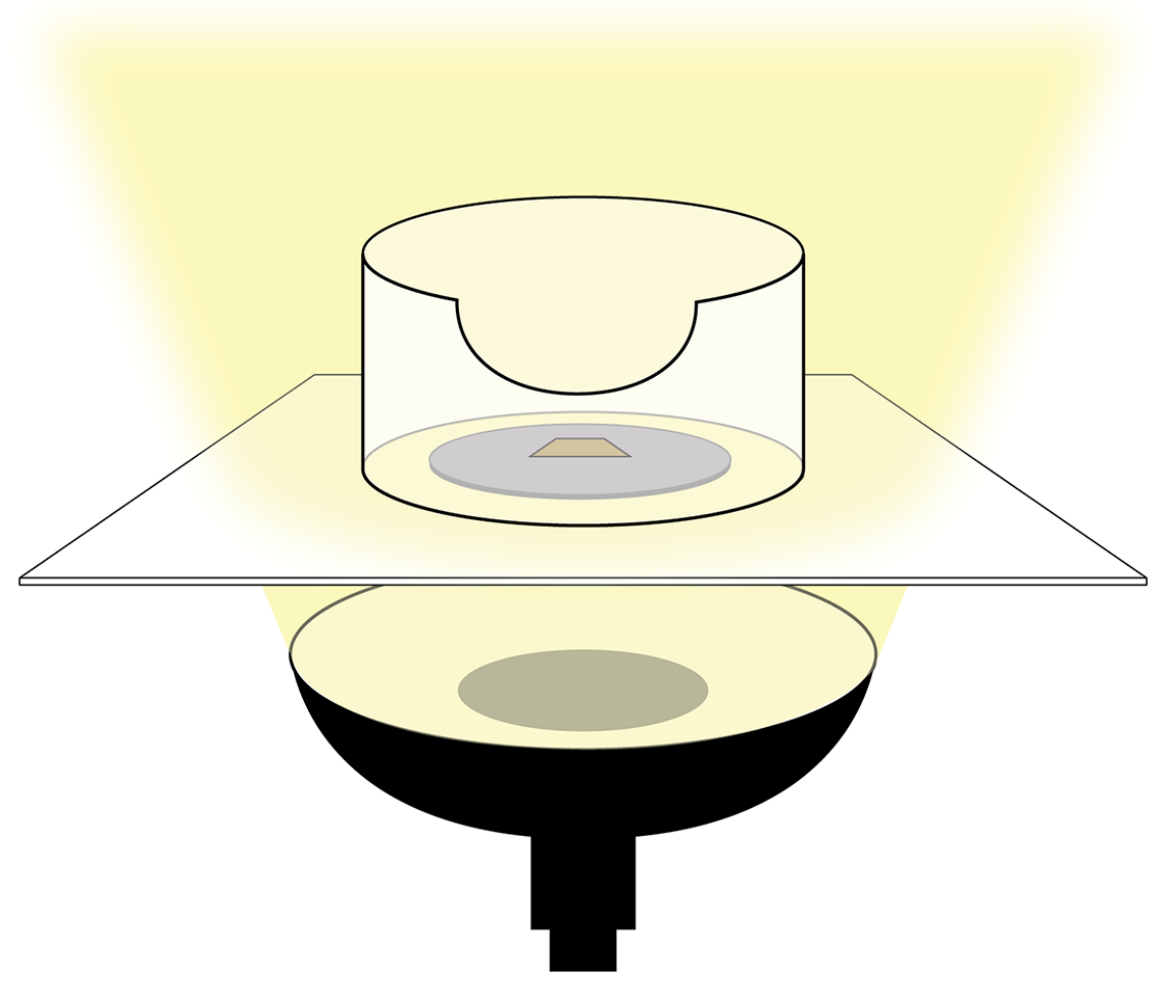

Dino-Lite AM4 I I 3 T

The urgency to verify the possibility of using cheaper and faster instrumentation than the one previously illustrated - as well as wanting to test its accuracy - prompted to compare the results obtained with macro photographic lens to those obtained with microscopic systems, in particular using a digital portable microscope, the Dino-Lite AM4I I3T. This instrument is part of the wide range of microscopes equipped with digital cameras used in recent years mainly for medical and industrial applications and applied to the field of cultural heritage [Esmaeili, Ebadi 2017, p. 65-67]. Their lightness, ease of use and ability to move, allows to obtain easily images, ensuring adequate intersection angles respect to the object. On the other hand, the narrow field and very limited depth of field represent obvious restrictions. The instrument used is characterized by a 1,3 MP photographic sensor $(1.280 \times 1.024 \mathrm{px})$, polarized light, a magnification factor of up to $200 \mathrm{x}$ and a tool for measuring linear distances; the cost of this equipment is around $€ 500$. Connecting the microscope to a portable station, through a dedicated procedure DinoCapture 2.0, 490 images were acquired at maximum resolution (3,75 MB Each), at an average operating distance of about $0,020 \mathrm{~m}$, with a $30 \times$ magnification factor. The images were acquired with a photogrammetric multi-image paradigm, thus ensuring an average overlap between the frames of about $70 \%$, manually moving the support of the tablet under the microscope secured on a stand (fig. 5). The operation lasted about 50 minutes, including the metric calibration operations for the chosen magnification factor.

The need to acquire a large number of images for the object's documentation derives from the fact that the digital microscope used is characterized, at the operating distance chosen for the acquisition, by a FOV of 13,0x 10,4 mm and a DOF of I,9 $\mathrm{mm}$. 
Fig. 4. Foil acquisition with 'macro' equipment; in the background: RRL lighting ring.

Fig. 5. Foil acquisition with Dino-Lite AM4I I 37.
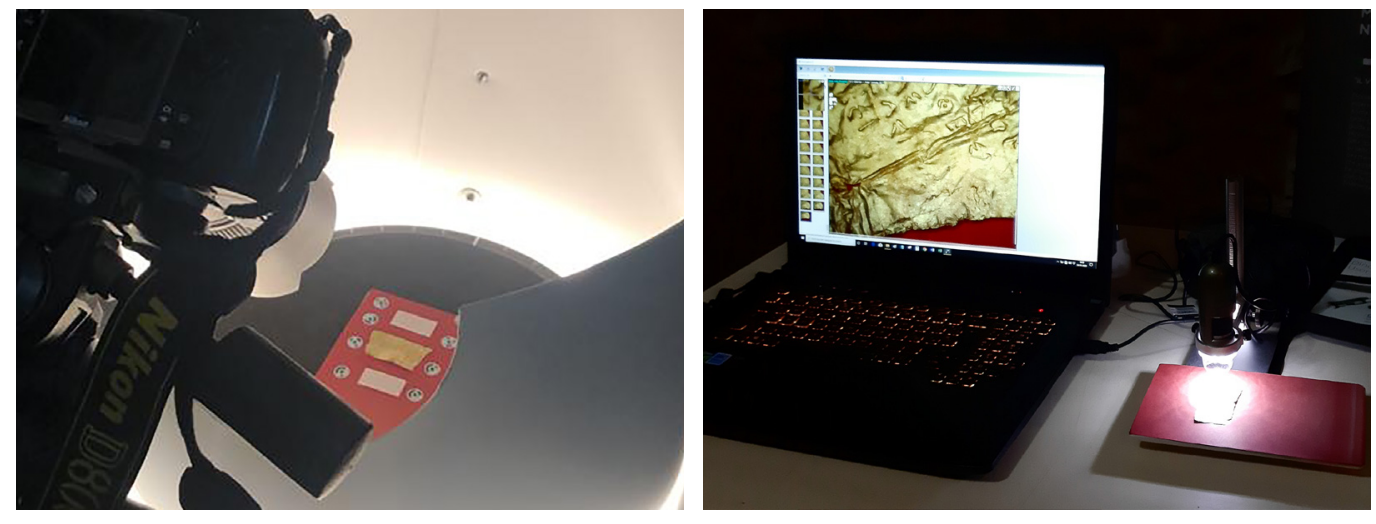

\section{Photogrammetric processing}

The two sets of images were processed in a SfM (Structure from motion) type software: RealityCapture. Both versions of the project were processed using the same PC equipped with Intel 19 9900k CPU, RTX2080ti GPU, 64 GB RAM. Both projects, with related temporary files, were placed on M.2 SSD. In RealityCapture the data processing is divided into three steps: Align Images (Max feature per image 70.000); Reconstruction (High detail); Texture. In the "macro" system dataset case the time taken to process I, I2 GB of images was about 50 minutes; before the photogrammetric process, the exposure of the images was optimized in Camera Raw and this process took around I5 minutes. In the "micro" system dataset case the time taken to process I,8 GB of images was about 60 minutes. At the end of the elaboration process, a textured mesh and an orthophoto were exported for both models. In both cases, the generation and export of the orthophoto took about 10 minutes. The mesh coming from the first system is made up of 6,7 million polygons, while the one made with Dino-Lite up of 19,8 (fig. 6). As for orthophotos, an orthophoto (fig. 7) composed by $8466 \times 604 \mathrm{I} \mathrm{px}\left(1,5 \cdot 10^{-3} \mathrm{~mm} / \mathrm{px}\right.$ ) was generated from the data obtained with photographic equipment, while from the data obtained with microscopic instrumentation, the orthophoto generated (fig. 8 ) is composed by $24808 \times 405$ I 8 $\mathrm{px}\left(7,8 \cdot 10^{-5} \mathrm{~mm} / \mathrm{px}\right)$.

\section{Data validation}

Data set up

The two models, using CloudCompare 2.10.2 open source application, were brought into real world units $(\mathrm{mm})$ through a direct scaling. In the 'macro' system, this operation was carried out by positioning two scale bars in the scene (one for scaling and one for checking the accuracy of the operation). In the second case, considering the extremely modest FOV of the Dino-Lite, which would have required the acquisition of a few hundred additional images to be able to include in the scene the scale bars, it was made by using the measuring tool present in DinoCapture 2.0. This tool, after generating a calibration profile for the magnification factor at the chosen distance, allows you to take sufficiently detailed flat measurements directly from the individual shots.

After restoring the correct scale factor for the two surfaces obtained, in order to be able to compare their descriptive quality, they were aligned through homologous points and an automatic alignment was carried out by an ICP algorithm (Iterative Closest Point) to refine the overlap. 
Fig. 6. Photogrammetric cloud and resulting mesh the 'micro' system. (red one) generated by
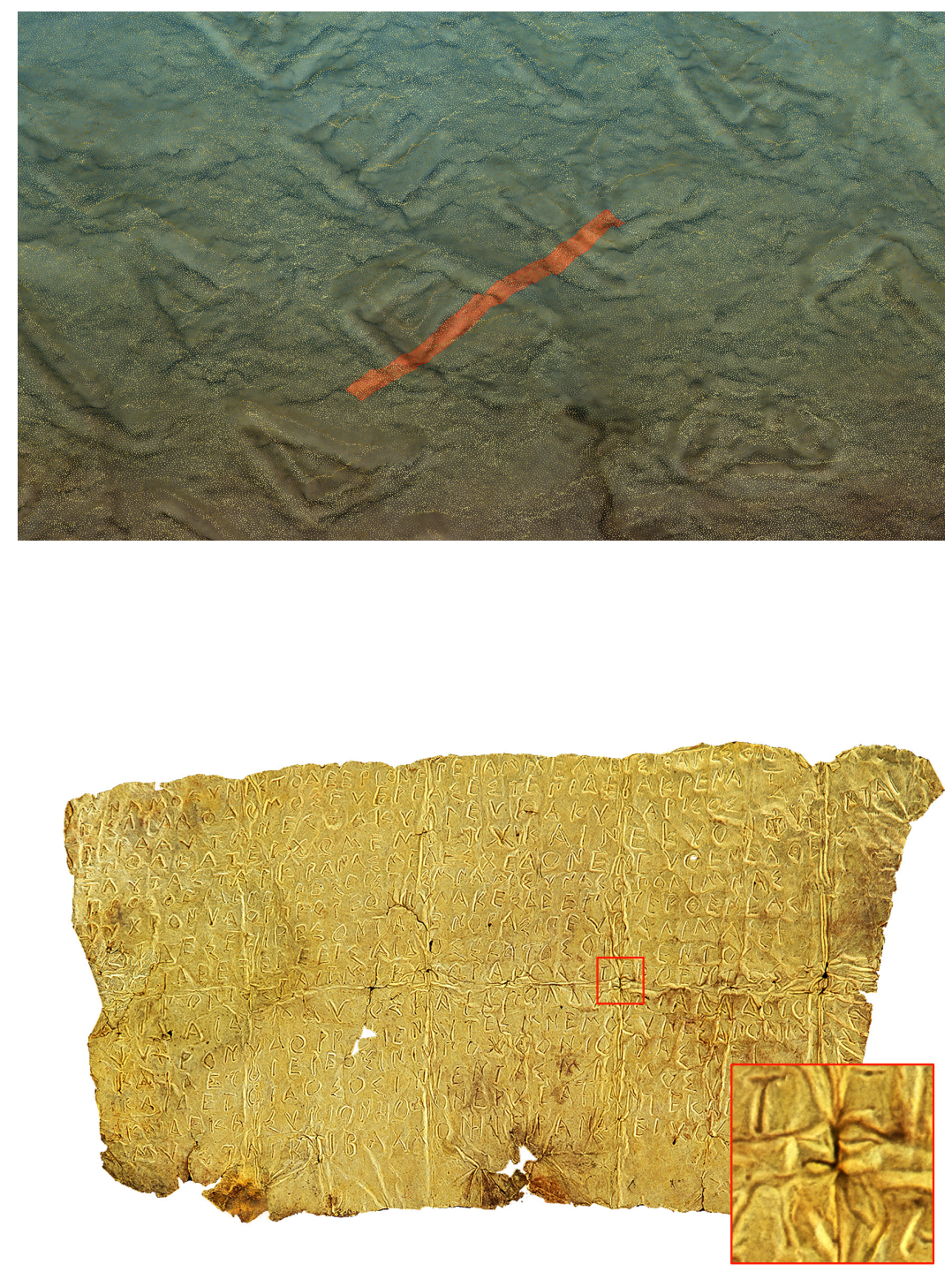
nerated with the datase from the photographic acquisition.

Fig. 8. Orthophotos generated with the datase from the microscopic acquisition.

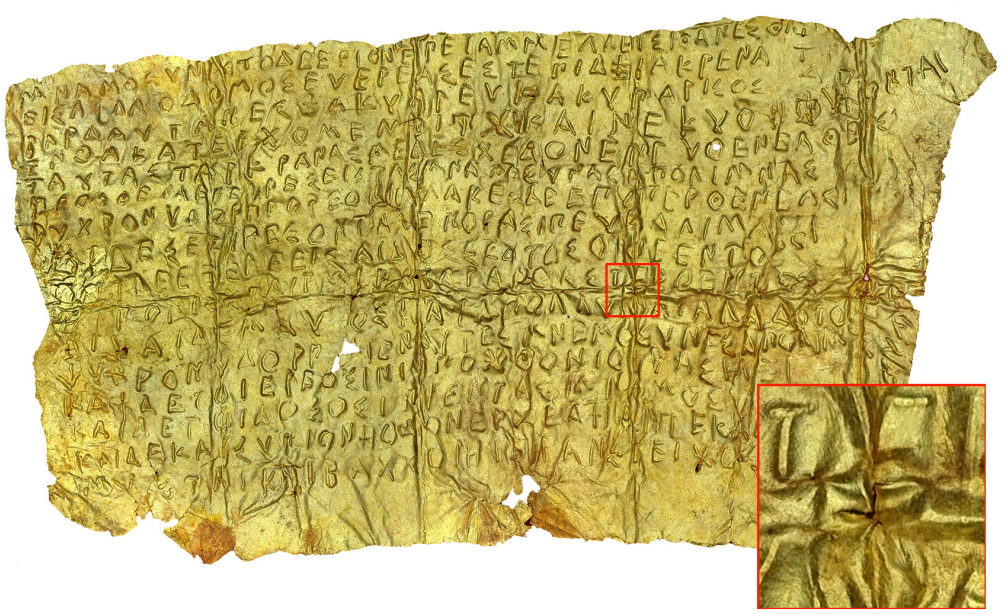




\section{Dataset analysis}

The evaluation phase of the surface's descriptive quality was carried out in open source software environment CloudCompare, using the C2C tool, which searches, for each point of the cloud or triangle of the mesh in phase of comparing, the one closest to the reference entity, thus defining an offset value of the first compared to the second. Using the cloud generated with the Dino-Lite as a reference, it was compared with 'macro' system cloud, highlighting the deviations of the surfaces between $-5 \mathrm{~mm}$ and $0 \mathrm{~mm}$ in false colors (fig. 9): the calculations show that the average deviations do not exceed $2 \mathrm{~mm}$.

Note also that the major deviations $(>5 \mathrm{~mm}$ ) are mainly present on the sides of the scene. This difference is due to the distance of these areas from the point of interest of the relief, caused by the choice to use a rotating table in the 'macro' photographic acquisition. In fact, it allowed to make a rapid succession of shots at a fixed distance from the subject, adjusting only the height of the camera and avoiding the repositioning of the tripod between each shot, but the need to frame the entire object for each shot has reduced the quality of the data significantly. The difference between the two models in the marginal areas is furthermore accentuated by the extremely close orthogonal capture scheme followed by the Dino-Lite, whose input data are therefore not affected by any significant loss of detail.

Fig. 9. Cloud to cloud comparison in a Cloud Compare environment for the evaluation of cloud deviations obtained with two different acquisition techniques.

Fig. 10. Comparison of color per vertex effect to the data generated with the 'micro' system, whith the 'micro' system, white with the 'macro' system.
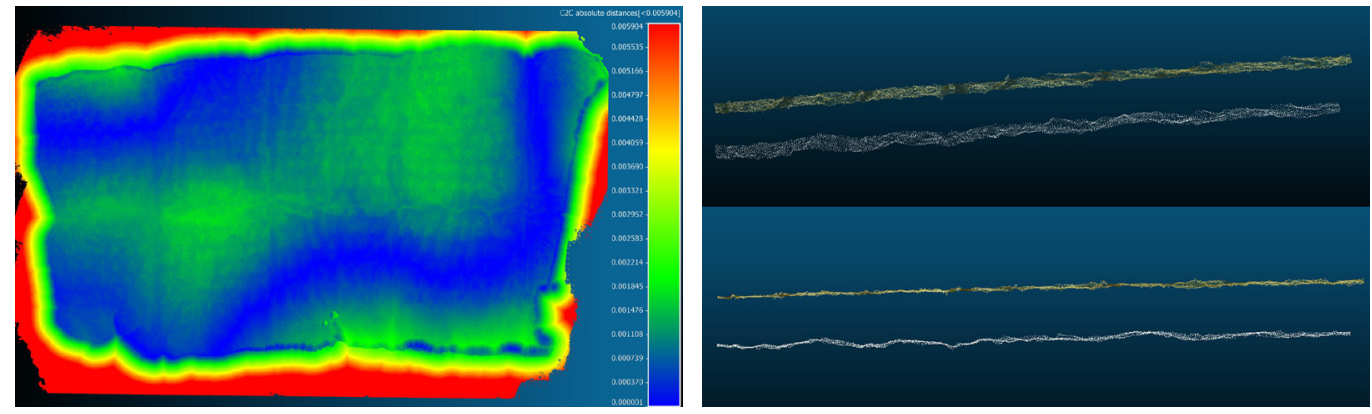

\section{First conclusions}

Overall dimensions of the equipment and cost-effectiveness, with a price that is $90 \%$ lower and the overall quality of the data, especially the two-dimensional one, suggest that the Dino-Lite AM4 I I 3T should be used as a solution for predominantly thin objects (fig. I I). As regards the three-dimensional description of the surface, there is a modest difference between the two systems, with a moderately better quality generated by the Dino-Lite AM4 I I3T data compared to the "macro" system data regarding to the description of the micro-incisions; on the contrary, the description of the general shape of the foil is definitely better in the survey obtained from the latter (fig. I0).

The result can be improved through the integration of the two techniques, which however would require long and cumbersome activities of recording the camera positions, in order to guarantee the goodness of the geometry obtained with the 'macro' system and costs and details of the 'micro' one.

Further experiments will be carried out by comparing the microscopic system with structured light equipment and photographic techniques aimed at obtaining a greater detail, by reducing the distance between the optical system and the object, using optical extension devices [5]. In this way, it will be possible to focus more closely on the subject, to have a greater magnification and to increase the detail of the acquisition, to knot the relationships and re-read the mystery history of this foil within the Visualizing Innovative and Social Artworks' project of Calabria region. 
Fig. II. In brief the main characteristics of the two acquisition systems implemented.

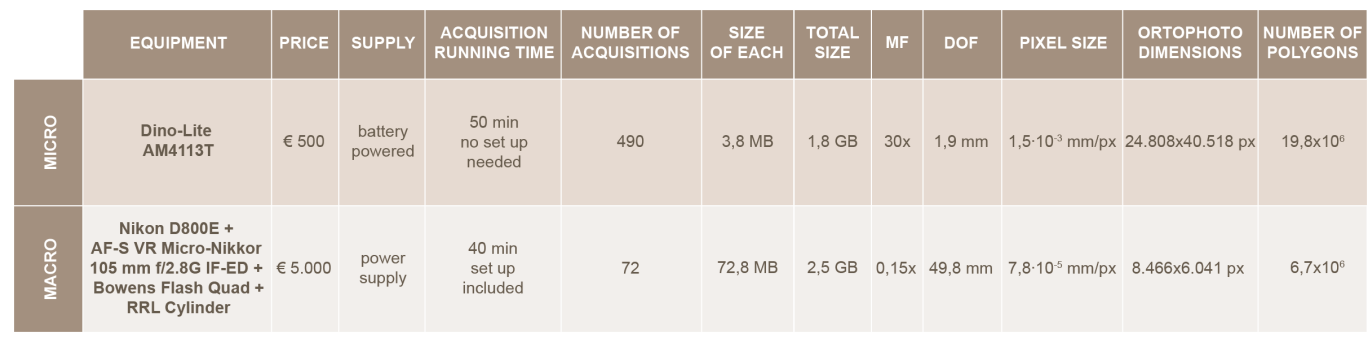

Notes

[I] The Orphic foil represents an important testimony of the cult of mystery religions in Calabria (perhaps already in use before the arrival of the Greeks). Placed, folded in four parts, on the chest of the deceased - who in the case was a girl of a noble family - it was intended to accompany her soul and guide her in the kingdom of Hades.

[2] The increase in exposure time is defined here as "relative" because it depends not only on the aperture, but also on the amount of radiant energy available in the unit of time, or the light power supplied by the artificial source. It seems trivial to observe that, with the same diaphragm and in continuous light conditions (such as outdoors), the time will be univocally determined by the value of this quantity, fixed a priori; under conditions of artificial flash light, however, it happens that the time can be determined by the operator, setting the generator power upstream.

[3] The explicit values derive from the mathematical formalization of the problem in the field of simplifying hypotheses introduced by geometric optics.

[4] Noise consists of an undesirable by-product of image capture that obscures the desired information, overlapping with a 'perfect' signal, made up of two simultaneous components: the luminance noise and the chrominance noise. The former is proportional to the ISO value, the latter is a function of exposure time.

[5] Optical system extension device positioned between the lens and the camera mount: by moving the lens away from the camera, the lens' front element will be closer to the subject, ensuring, despite this, the focus.

\section{References}

Bolognesi Cecilia Maria, Fiorillo Fausta (2018). Optimization of texture mapping process in the Reality-Based Modeling application. In Salerno Rossella (a cura di). Rappresentazione/Materiale/lmmateriale. $40^{\circ}$ Convegno internazionale dei Docenti delle Discipline della Rappresentazione. Milano 15- 17 Settembre 20 18. Roma: Gangemi Editore International, pp. 337-342.

Greenleaf Allen R. (1950). Photographic optics. New York: Macmillan.

García Fernández Jorge, Álvaro Tordesillas Antonio, Barba Salvatore (20I5). An approach to 3D digital modeling of surfaces with poor texture by range imaging techniques. 'Shape from stereo' vs. 'Shape from silhouette' in digitizing Jorge Oteiza's sculptures. In The International Archives of the Photogrammetry, Remote Sensing and Spatial Information Sciences, Volume XL-5/ W4, pp. 25-29. 3D Virtual Reconstruction and Visualization of Complex Architectures, 25-27 February, Avila, Spain.

Esmaeili Farid, Ebadi Hamid (2017). Handy microscopic close-range videogrammetry. In The International Archives of the Photogrammetry, Remote Sensing and Spatial Information Sciences, Volume XLII-4M4, 2017, pp. 65-67. Tehran's Joint ISPRS Conferences of GI Research, SMPR and EOEC 2017, 7-10 October 2017.

Marshall Matt, Johnson Andrew, Summerskill Steve, Baird Quinner, Esteban Enrique (2019). Automating photogrammetry for the 3d digitisation of small artefact collections. In The International Archives of the Photogrammetry, Remote Sensing and Spatial Information Sciences, Volume XLII-2N I 5, 2019, pp. 75 I-757. 27th CIPA International Symposium "Documenting the past for a better future", I-5 September, Ávila, Spain.

Nicolae Corina, Nocerino Erica, Menna Fabio, Remondino Fabio (20 I4). Photogrammetry applied to problematic artefacts. In The International Archives of the Photogrammetry, Remote Sensing and Spatial Information Sciences, Volume XL-5, pp. 45 I-456. In ISPRS Technical Commission V Symposium, 23-25 june, Riva del Garda, Italy.

Pugliese Carratelli Giovanni (200 I). Le lamine d'oro orfiche. Istruzioni per il viaggio oltremondano degli iniziati greci. Milano: Adelphi.

Russo Michele (2019). Fotomodellazione 2020: viaggio di sola andata? In Belardi Paolo (a cura di). Riflessioni, l'arte del disegno/ il disegno dell'arte. $41^{\circ}$ Convegno internazionale dei docenti delle discipline della Rappresentazione. Perugia I9-2I settembre 20 19. Roma: Gangemi Editore International, pp. 425-430.

Sapirstein Philip (2018). A high-precision photogrammetric recording system for small artifacts. In Journal of Cultural Heritage, 3I, pp. 33-45.

\section{Authors}

Sara Antinozzi, Università degli Studi di Salerno, santinozzi@unisa.it

Diego Ronchi, Spiron Heritage and Survey, Roma, diego.ronchi@spiron.it

Salvatore Barba, Università degli Studi di Salerno, sbarba@unisa

To cite this chapter. Sara Antinozzi, Diego Ronchi, Salvatore Barba (2020). Macro e micro fotogrammetria per la virtualizzazione della laminetta orfica (V-IV a.C.) del Museo Nazionale di Vibo Valentia/Macro and Micro Photogrammetry for the Virtualization of the Orphic Foil (V-IV B.C) of National Museum of Vibo Valentia. In Arena A., Arena M., Brandolino R.G., Colistra D., Ginex G., Mediati D., Nucifora S., Raffa P. (a cura di). Connettere. Un disegno per annodare e tessere. Atti del $42^{\circ}$ Convegno Internazionale dei Docenti delle Discipline della Rappresentazione/Connecting. Drawing for weaving relationships. Proceedings of the 42th International Conference of Representation Disciplines Teachers. Milano: FrancoAngeli, pp. $1538-1555$ 